Oral

\title{
Sperm DNA Fragmentation Among Smokers and Non-Smokers as Measured by Sperm Chromatin Dispersion Test
}

\author{
Azha Syahril Azizan ${ }^{1}$, Kamarul Bahyah Mustafa ${ }^{1}$, Azantee Yazmie Abdul Wahab ${ }^{1}$, \\ Roszaman Ramli ${ }^{1}$, Nurkhairulnisa Abu Ishak ${ }^{1}$ \\ ${ }^{1}$ Department of Obstetric and Gynaecology, Kulliyyah of Medicine, International \\ Islamic University Malaysia
}

Presenter: Azha Syahril Azizan

Introduction: Traditionally, seminal fluid analysis is done to assess male infertility particularly sperm concentration, morphology and motility. Sperm chromatin dispersion test explores the quality of the sperm DNA structures. High sperm DNA fragmentation is associated with unexplained infertility, failure of assisted reproduction and recurrent miscarriages. Smoking has been associated with high sperm DNA fragmentation in some studies but not in others. Materials and Methods:

A comparative cross-sectional study, involving 32 smokers and 32 non-smokers to determine the association between cigarette smoking and sperm DNA fragmentation. Semen samples were collected from patients undergoing seminal fluid analysis (SFA) at the IIUM Fertility Centre and IIUM Medical Centre O\&G Clinic from January 2017 to June 2018. Sperm chromatin dispersion test was done and level of $15 \%$ is considered upper limit of normal. Results: $53.1 \%$ in the smoker group have abnormal level of sperm DNA fragmentation compared to $34.4 \%$ of nonsmokers. The median and interquartile range for smokers were 15.30 and 19.0 (10.425 - 29.375) respectively while for non-smokers were 8.25 and 17.8 (4.075 $21.850)$ which was statistically significant $(p=0.012)$. It was also noted that three participants in the smoker group (9.4\%) has very high sperm DNA fragmentation index of more than $80 \%$ even though they have normal seminal fluid analysis. Conclusion: There is an association between smoking and high sperm DNA fragmentation. There is a trend of increased in DNA fragmentation in smokers even though their SFA results were normal. 